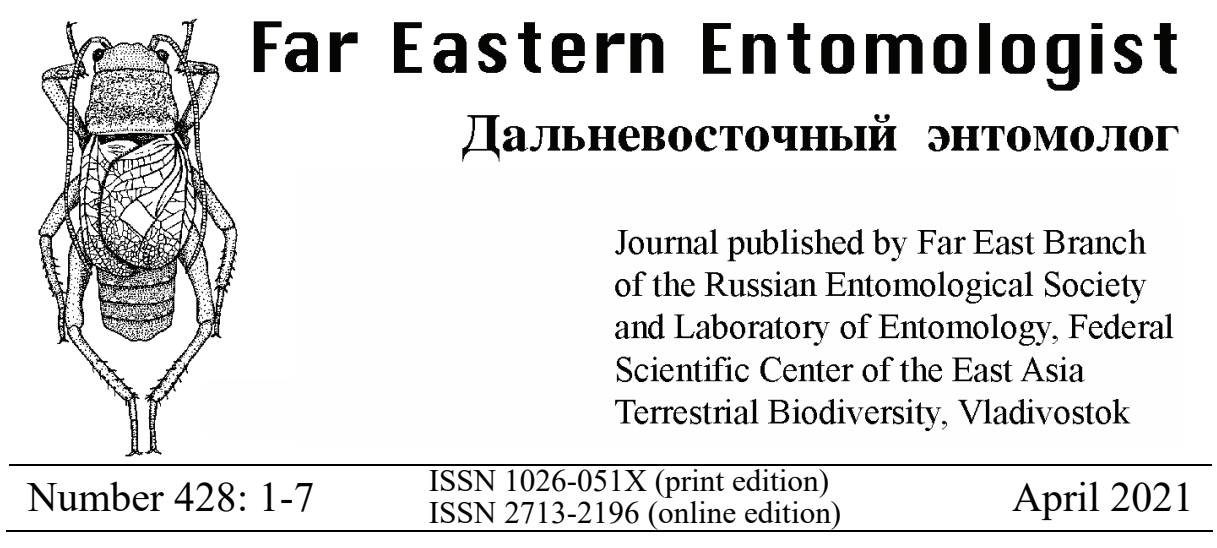

https://doi.org/10.25221/fee.428.1

http://zoobank.org/References/DC39CD56-ED26-4A2D-860A-1D844FD59A26

\title{
FIRST RECORD OF THE GENUS ZAOMMOMENTEDON GIRAULT, 1915 (HYMENOPTERA: EULOPHIDAE) FROM INDIA, WITH DESCRIPTION OF A NEW SPECIES
}

\author{
M. M. Jamali ${ }^{1, *)}$, S. B. Zeya ${ }^{2)}$
}

1) School of Agricultural Sciences, Glocal University, Saharanpur 247121, Uttar Pradesh, India. *Corresponding author, E-mail: majidjamali1988@gmail.com

2) Department of Zoology, Aligarh Muslim University, Aligarh 202 002, Uttar Pradesh, India.

Summary. The genus Zaommomentedon (Chalcidoidea: Eulophidae) is recorded for the first time from India. A new species Z. giraulti Jamali et Zeya, sp. n. is described from Andhra Pradesh, India.

Key words: Chalcidoidea, Entedoninae, Zaommomentedon, taxonomy, new species, fauna, new record, Andhra Pradesh, India.

М. М. Джамали, С. Б. Зея. Первое указание рода Zaommomentedon Girault, 1915 (Нуmenoptera: Eulophidae) из Индии с описанием нового вида // Дальневосточный энтомолог. 2021. N 428. С. 1-7.

Резюме. Впервые для Индии указан род Zaommomentedon (Chalcidoidea: Eulophidae). Из штата Андхра-Прадеш описан Z. giraulti Jamali et Zeya, sp. n.

\section{INTRODUCTION}

The genus Zaommomentedon was erected by Girault (1915) for the single species Z. mandibularis Girault, 1915. It is a little-known genus, containing only 
five species, namely Z. brevipetiolatus Kamijo, 1990, Z. mandibularis Girault, 1915, Z. milletiae (Kerrich, 1969), Z. nepticulae (Hedqvist, 1976), and Z. newbyi (Kerrich, 1969) (Girault, 1915; Kerrich, 1969; Hedqvist, 1976; Kamijo, 1990; Noyes, 2020). So far, no species of the genus have been known from India.

In this paper, we record the genus Zaommomentedon from India for the first time and describe a new species. A brief diagnosis of the genus is also given.

\section{MATERIAL AND METHODS}

The present study is based on specimens collected mainly by sweep net from Indian States of Andhra Pradesh. Body colour was noted from card mounted specimens before clearing and mounting the specimens on slides in Canada balsam. Body length for the new species is given in millimetres. All other measurements are relative taken from the divisions of a linear scale of a micrometer placed in the eye piece of a compound microscope. These measurements were taken at $100 \times$ magnification of the microscope.

The photographs of card mounted specimens were taken with a digital camera (Nikon DS-Fi2) attached to a stereo-zoom (Nikon SMZ25) and the photographs of slide mounted body parts were taken with a digital camera (Nikon DS-Filc) attached to a compound microscope (Nikon Eclipse Ci).

The following abbreviations are used in the text: $\mathrm{C} 1, \mathrm{C} 2$, etc. - clava segments 1st, 2nd, etc.; F1, F2, etc. - funicle segments 1st, 2nd, etc.

Holotype and paratypes of a new species are deposited at the Insect collections, Department of Zoology, Aligarh Muslim University, Aligarh, India (ZDAMU).

\section{TAXONOMY}

\section{Family Eulophidae Westwood, 1829}

\section{Subfamily Entedoninae Foerster, 1856}

\section{Genus Zaommomentedon Girault, 1915}

Zaommomentedon Girault, 1915: 187. Type species: Zaommomentedon mandibularis Girault, 1915, by original designation.

Visnuella Hedqvist, 1976: 51. Type species: Visnuella nepticulae Hedqvist, 1976, by original designation. Synonymized by Kamijo, 1990: 816.

DIAGNOSIS. FEMALE. Head broader than mesosoma; mandible with 6 small teeth (Fig. 5); eye large and pubescent, covering almost complete height of head. Antenna with funicle 3-segmented and clava 2-segmented; last claval segment with a long spicula. Pronotum collar with a sharp margin; notauli well developed; mesoscutum and scutellum smooth, both with a conspicuous median groove (Figs 7, 8); propodeum smooth, without median carina; postmarginal vein longer than stigmal vein; gaster short and ovate with elongate petiole (Fig. 9). MALE. Scape short and broad; flagellum has much longer pilosity than in the female (Fig. 10). 
COMPOSITION. Until now this genus includes five species distributed in Congo, Nigeria, Israel, Sri Lanka, Taiwan, Thailand, Japan, Papua New Guinea, and Australia. One new species from India is described below.
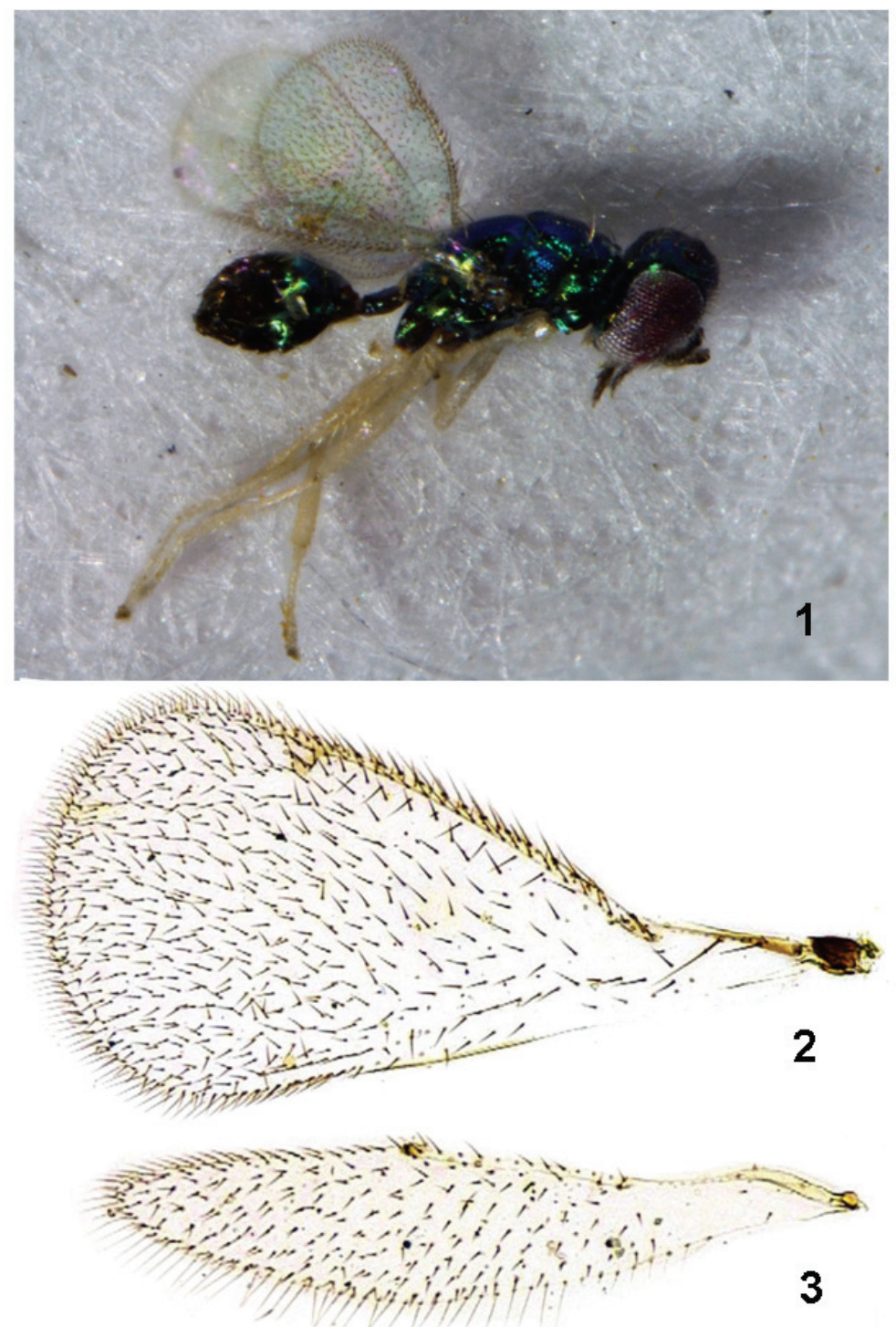

Figs 1-3. Zaommomentedon giraulti sp. n., holotype, female. 1 - habitus; 2 - fore wing; 3 - hind wing. 
Zaommomentedon giraulti Jamali et Zeya, sp. n.

http://zoobank.org/NomenclaturalActs/067040D1-FAF5-486B-9076-20A86C09EEEC

Figs 1-10

TYPE MATERIAL. Holotype - female (on slide under 4 coverslips, slide No. 109), India: Andhra Pradesh, Guntur, Kolanukonda, 11.II 2014, coll. M.T. Khan (ZDAMU). Paratypes: 2 females (on slides, slide Nos. EUL.86, EUL.108), 2 males (on slides, slide Nos. EUL.27, EUL.108), India: Andhra Pradesh, Guntur, Rajmandi, 11.II 2014, coll. M.T. Khan (ZDAMU).

DESCRIPTION. FEMALE (Fig. 1). Holotype. Body length $1.04 \mathrm{~mm}$. Head metallic green. Antenna with scape white; pedicle, F1 and F2 dark brown, F3 and clava brown. Mesosoma metallic green with bluish reflection except pronotum dark brown. Wings hyaline. All legs pale white except hind coxae completely and mid coxae in basal half brown. Metasoma with petiole dark brown, gaster metallic green.

Head (Fig. 4). Head in front view, $1.37 \times$ as broad as long; upper frons with alveolate reticulation and lower with transverse reticulation; eye height $12.5 \times$ as long as malar space; antennal toruli situated above the lower eye margin. Antenna (Fig. 6) with scape $5.4 \times$ as long as broad, $3 \times$ as long as pedicel; pedicel $1.5 \times$ as long as broad, shorter than all funicle segments individually; pedicel + flagellum $0.83 \times$ head width; funicle 3-segmented, all funicle segments subequal in length with longitudinal sensilla; clava 2 -segmented, $1.46 \times$ as long as broad; last claval segment narrow.

Mesosoma (Figs 7, 8). Mesosoma $2.14 \times$ as long as broad; pronotum narrow with six long setae on posterior margin; mesoscutum slightly shorter than scutellum; mid lobe of mesoscutum with 4 long setae and a deep median groove extending nearly half length of mesoscutum anteriorly; notauli posteriorly faint and indistinct; scutellum subquadrate, smooth with 2 setae and a median longitudinal groove touching anterior and posterior margins. Fore wing (Fig. 2) $1.91 \times$ as long as broad with 9 admarginal setae; marginal vein + parastigma $2.25 \times$ as long as submarginal vein, $14.4 \times$ as long as stigmal vein; post marginal vein $2 \times$ as long as stigmal vein; longest marginal seta $0.11 \times$ maximum wing width. Hind wing (Fig. 3) $4.6 \times$ as long as broad; longest marginal seta $0.34 \times$ maximum wing width

Metasoma (Fig. 9). Petiole $0.73 \times$ as broad as long, broadened medially with a teeth like projection on both side; gaster shorter than mesosoma; ovipositor occupying half length of gaster, not protruded beyond apex of gaster and $0.86 \times$ as long as hind tibia.

Relative measurements (holotype). Head height: width, 27: 37; eye height, 25; malar space, 2. Antennal segments length: width-scape, 13.5: 2.5; pedicel, 4.5: 3; F1, 5.5: 3.5; F2, 5: 3.5; F3, 5.25: 3; C1, 4.25: 3.5, C2, 3.5: 2.25. Mesosoma length: width (dorsal view), 46: 31.5. Fore wing length: width, 69: 36; longest marginal seta, 4; submarginal vein length, 16; parastigma length, 3; marginal vein length, 33; stigmal vein length, 2.5; postmarginal vein length, 5. Hind wing length: width, 60: 13; longest marginal seta, 4.5; hind tibia , 23. Petiole length: width, 8.5: 6; gaster length, 42; ovipositor length, 20. 


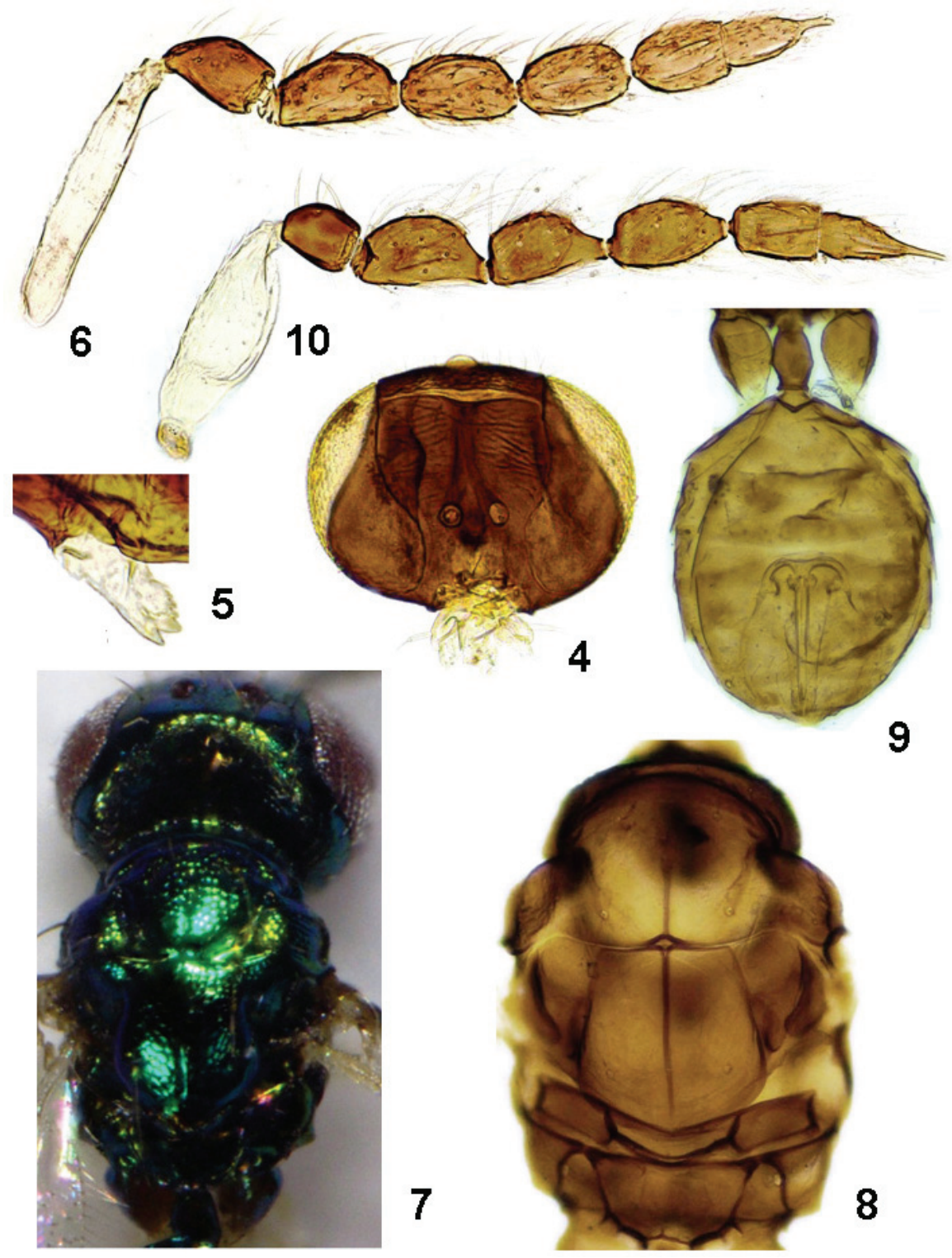

Figs 4-10. Zaommomentedon giraulti sp. n. 4-9 - holotype female: 4 - head, frontal view; 5 - mandible; 6 - antenna; 7 - mesosoma with head, dorsal view; 8 - mesosoma; 9 metasoma with petiole; 10 - paratype male, antenna. 
Table 1. Morphological differences between Zaommomentedon giraulti sp. n. and Z. brevipetiolatus Kamijo, 1990

\begin{tabular}{l|l|l}
\hline Characters & Zaommomentedon giraulti sp. n. & Zaommomentedon brevipetiolatus \\
\hline Mesosoma & $2.14 \times$ as long as broad & $1.6 \times$ as long as broad \\
\hline $\begin{array}{l}\text { Median } \\
\text { groove }\end{array}$ & $\begin{array}{l}\text { extending up to half length of } \\
\text { mesoscutum anteriorly }\end{array}$ & $\begin{array}{l}\text { extending up to three-fourth of } \\
\text { mesoscutum anteriorly }\end{array}$ \\
\hline Scutellum & $\begin{array}{l}\text { either subquadrate or a little broader } \\
\text { than long, without longitudinal } \\
\text { sculpture }\end{array}$ & $\begin{array}{l}\text { slightly longer than broad, with } \\
\text { longitudinal sculpture }\end{array}$ \\
\hline Propodeum & $3 \times$ as long as dorsellum & $2 \times$ as long as dorsellum \\
\hline $\begin{array}{l}\text { Marginal vein } \\
+ \text { parastigma }\end{array}$ & sub equal to wing width & distinctly longer than wing width \\
\hline $\begin{array}{l}\text { Postmarginal } \\
\text { vein }\end{array}$ & $2 \times$ as long as stigmal vein & $3 \times$ as long as stigmal vein \\
\hline Gaster & slightly longer than thorax & slightly shorter than thorax \\
\hline
\end{tabular}

MALE. Similar to female in body colouration. Antenna (Fig. 10) with scape $2.6 \times$ as long as broad, relatively broad; funicle segments dorsally curved, narrower apically; eye short and malar space long.

Relative measurements (paratype). Head height: width, 27: 38; eye height, 20; malar space, 6. Antennal segments length: width- scape, 13: 5; pedicel: 4: 3; F1, 7.5: 4.25; F2, 7: 4; F3, 7: 4; C1, 5.25: 3.5, C2, 5.5: 2.75 .

DIAGNOSIS. The new species is closely related to Z. brevipetiolatus Kamijo, 1990 but differs from latter in characters given in Table 1.

HOST. Unknown.

DISTRIBUTION. India: Andhra Pradesh.

ETYMOLOGY. The species is named after the name of Alexandre Arsène Girault for his contribution to the chalcids taxonomy.

\section{ACKNOWLEDGEMENTS}

We thank the Chairman, Department of Zoology, Aligarh Muslim University, Aligarh, for providing research facilities. Thanks are due to Dr. Mohammad Hayat, the Principal Investigator of the ICAR "Network Project on Insect Biosystematics" AMU Centre, Department of Zoology, Aligarh Muslim University, Aligarh, for constant help and encouragements. We are also grateful to Dr. Rayees Afzal Mir, Glocal University, Saharanpur, for his valuable suggestions.

\section{REFERENCES}

Girault, A.A. 1915. Australian Hymenoptera Chalcidoidea IV. Supplement. Memoirs of the Queensland Museum, 3: 180-299.

Hedqvist, K.J. 1976. Descriptions of new chalcid flies (Hym., Chalcidoidea, Encyrtidae and Eulophidae) reared from Nepticula species collected in Sri Lanka. Entomologisk Tidskrift, 97(1-2): 50-54. 
Kamijo, K. 1990. Notes on Pleurotropposis (Hymenoptera, Eulophidae) and its allied genera, with descriptions of four new species from Japan. Japanese Journal of Entomology, 58: 816-826.

Kerrich, G.J. 1969. Systematic studies on eulophid parasites (Hym., Chalcidoidea), mostly of coffee leaf-miners in Africa. Bulletin of Entomological Research, 59(2): 222-223.

Noyes, J.S. 2020. Universal Chalcidoidea Database. World Wide Web electronic publication. Avaliable at: http://www.nhm.ac.uk/chalcidoids/ (Accessed: 8 September 2020). 